\title{
Windows are less glaring when there is a preferred view
}

\author{
Nuanwan Tuaycharoen
}

\begin{abstract}
This study aims to investigate the effects of view content as related to landscape preference on the sensation of discomfort. The study began with an investigation of the effect of the presence of a view on discomfort glare. The experiment was carried out in a laboratory investigating real views with 32 students as subjects. Following this, the effects of four factors related to landscape preference were investigated: complexity, coherence, mystery, and legibility. This study was done in a laboratory using small projected images representing real scenes with 24 subjects. The last experiment aimed to see the effect of view complexity and mystery. This was carried out in a real school classroom using 32 Thai students as subjects. The result showed that there is an important effect from the presence of a view on discomfort glare. The findings also suggested several significant effects on the sensation of glare discomfort, for example, view mystery, and view complexity.
\end{abstract}

Keywords: Discomfort glare, view, landscape preference, daylighting

\section{Introduction}

Discomfort glare is the annoyance, or temporary discomfort produced by luminance within the visual field that is sufficiently greater than the luminance to which the eyes are adapted. Presently, existing small source glare formulae are often poor predictors of the subjective assessment of discomfort glare (Manabe, 1976; Boyce, 1981). This suggests that discomfort glare depends on more factors than the four embodied in the glare calculations: source luminance, source size, surround luminance, and position index.

Some evidence of discomfort glare from windows suggested the importance of view on the sensation of discomfort. One of the most important characteristics of window-views is their horizontal stratification (Markus, 1976a). In the field of landscape aesthetics, a pioneering study in scenic-quality presented a landscape preference model based upon four important variables: complexity, coherence, mystery, and legibility.

The present study aims to investigate the effects of view content as related to landscape preference on the sensation of discomfort. There are three experiments in this study. The study began with an investigation of the effect of the presence of view on discomfort glare. The experiment was carried out in a laboratory investigating real views with 32 students as subjects. Following this, the effects of four factors related to landscape preference were investigated: image complexity, coherence, mystery, and legibility. The study was done in a laboratory using small projected images representing real scenes with 24 subjects. The last experiment aimed to see the effect of view complexity and mystery. This was carried out in a real school classroom using 32 Thai students as subjects.

\section{Experiment I: The Effect of Presence of View on Glare}

The first experiment aimed to see the effect of the presence of view on glare. This study was carried out in a laboratory investigating glare from a windowview.

\section{Experimental Setting}

The experiments were conducted in the laboratories of the School of Architecture, the University of Sheffield. Subjects sat in an open cubicle facing a view that could be seen through an aperture, which acted as a glare source. The cubicle was half-hexagonal in plan; the interior surfaces were painted matt white internally. Stray light outside was masked. Subjects were positioned with their eyes at the centre of the opening. The size of the opening was $25 \times 25 \mathrm{~cm}$. One fixation point was used - the centre of the righthand opening. Background luminance was provided by reflecting light off the surfaces in the subject's field of view. A luminaire connected with a dimmable transformer to adjust the light for source luminance was held constant for each person.

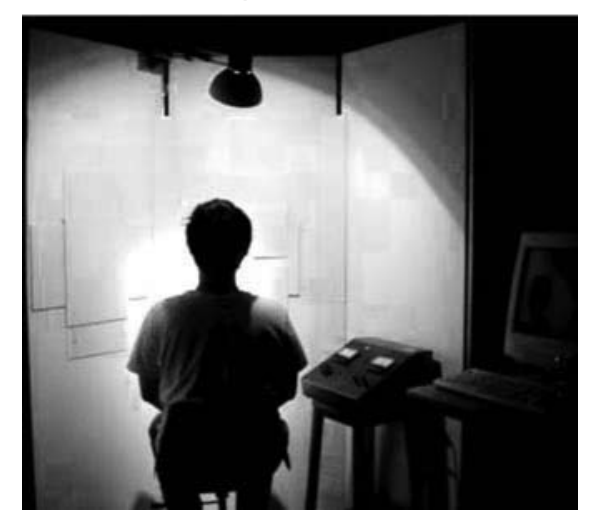

Figure 1: View of the experimental setting 


\section{Experimental Procedure}

Thirty-two subjects were involved in this experiment. All of them were university students: 16 men and 16 women, aged 18-30. Spectacles were worn by $50 \%$ of both the men and women, but no other eye defects or no colour-blindness was reported by any subject in this experiment. There were two treatments in this experiment. Treatment 1 used a real view, and treatment 2 used a blank screen representing a no-view condition.

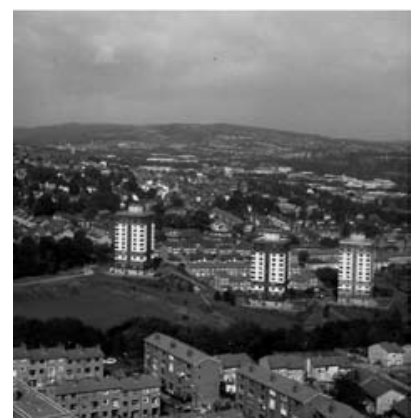

Figure 2: Treatment 1: a view (left) and treatment 2:a blank screen (right)

The experimental design was a repeated measures Balanced Latin Square design with two-treatment conditions. By using this design, the effects due to nuisance variables from subjects and people involved in the experiment or due to fatigue could be counterbalanced. There were two periods in this experimentthe presetting-up period and the real experiment. In this presetting-up period, each subject was first provided with an explanation of the study, informed consent form, and a pre-study questionnaire. The subject was then positioned in the chair and the appropriate instructions were read to the subject. The experimenter showed the subject an example of evaluating dis- comfort sensation between a real view seen through an opening and then the opening covered with by a blank translucent board. After that, the subject was asked to do one trial. Finally, the subject was allowed to relax for 10 minutes. After the presetting- up period, the real experiment was begun. First, the presenter asked the subject to look at the center of the opening containing an outside view (the first treatment). Then, the presenter instructed the subject to continue to look at the center and he/she was asked to evaluate the discomfort sensation by marking on the VAS and, at the same time, send a signal to the experimenter. The illuminance level of the view seen through the opening was recorded by the experimenter. Then, the experimenter closed the aperture, covered the view outside with the blank uniform board, and adjusted the light from the above luminaire to reach the same illuminance level as the first treatment. This period took about 2 minutes or until the subject indicated the afterimage effect was gone. The subject was, then, asked to fix his/her eyes at the center of the blank screen and to judge the glare from this uniform source by marking on the VAS again. Tests were continued until completing 32 subjects.

\section{Subjective Assessment of Discomfort Glare}

We used a visual analogue scale (VAS), an approach similar to that employed by several researchers, to evaluate glare in this study (Berman et al, 1996; Osterhaus and Bailey, 1992). The experimenter instructed subject to place the line or check mark on the scale to indicate his/her perceived level of discomfort. The subject's marks on the VAS were identified to the nearest millimetre with values ranging from 0 to 100 . The subjective glare ratings were related to the following numerical scale:

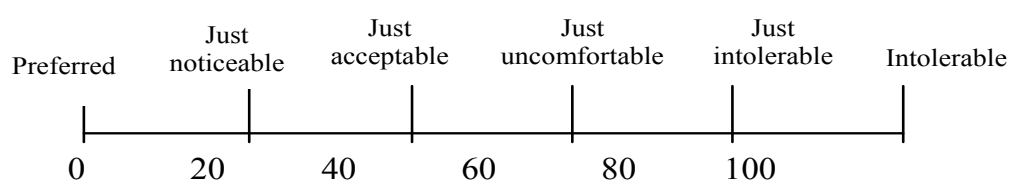

$0=$ preferred (comfortable) lighting conditions

$20=$ borderline between imperceptible and noticeable (just noticeable)

$40=$ borderline between noticeable and acceptable (just acceptable)

$60=$ borderline between acceptable and uncomfortable (just intolerable)

$80=$ borderline between uncomfortable and intolerable (just intolerable)

$100=$ intolerable

Figure 3: Visual analog scale (VAS) used in the subjective assessment of discomfort glare from windows

\section{Results}

Table 1: Mean and standard deviation of glare assessments (VAS) from two treatments and the significance levels ( $p$-value) of the Paired sample t-test

\begin{tabular}{lcc}
\hline Image categories & Mean & SD \\
\hline uniform blank screen & 46.94 & 4.87 \\
view & 20.91 & 2.00 \\
\hline$p$-value & $0.00 * *$ \\
\hline$* \quad$ The mean difference is highly significant $($ prob $<0.01)$ in a paired $t$-test & \\
$* \quad$ The mean difference is significant $(p r o b<0.05)$ in a paired $t$-test & \\
NS No significant difference in a paired $t$-test
\end{tabular}


Table 1 shows the mean and standard deviation of glare assessments (VAS) from two treatments and the significance levels ( $p$-value) of paired sample t-test analysis. The t-test indicates the effect of the presence of a view was highly significant $(p<0.01)$. In conclusion, the results in this experiment suggested that the presence of a view can reduce the degree of discomfort glare.

\section{Experiment II: The Effect of Image Character- istics on Discomfort Glare}

Tuaychareoen and Tregenza (2006) found that an interesting image can reduce the glare discomfort. There is also some evidence suggesting a relation between interest and preference (Tuaycharoen, 2006). $\mathrm{R}$. and S. Kaplan pioneered research into landscape aesthetics developing a landscape preference model based upon four important variables: complexity, coherence, mystery, and legibility. They pointed out that complexity offers enough information to promote interest. Ulrich (1977) restated Kaplan and Kaplan's findings as he argued that a visual landscape preference is a response in favour of a scene which relates to two main factors: legibility and mystery. In his terms, legibility has four components: complexity, focality (coherence and unity), ground texture, and depth. All of the evidence shows the possible effects of the four factors relating to the landscape preference on glare.

\section{Research Hypothesis}

The hypotheses for this part of the study are as follows:

1. An increase in complexity in an image leads to a decrease in discomfort glare

2. An increase in mystery in an image leads to a decrease in discomfort glare

3. An increase in image coherence in an image leads to a decrease in discomfort glare

4. An increase in image legibility leads to a de crease in discomfort glare

\section{Research Methodology Experimental Settings}

The experiments were conducted in the laboratories of the School of Architecture, Naresuan University. Subjects sat in an open cubicle similar to the one in the first experiment. There were three potential glare sources: a small diffuse source seen through an aperture, which acted as a reference, and two diffusing screens onto which images were back-projected. The cubicle was half-hexagonal in plan; the interior surfaces were painted matt white internally and illuminated from outside the cubicle to maintain a constant luminance distribution. The projected image size was $8 \times 8 \mathrm{~cm}$. One fixation point was used- the centre of the right-hand screen. For the test glare sources, two modified digital projectors located behind the partition projected images on diffusing screens. The projectors used 150W metal halide lamps, and the beams were condensed onto small areas with additional convex lenses. The projectors were connected to two computers which generated the images. The apparatus is shown in Figure 4.

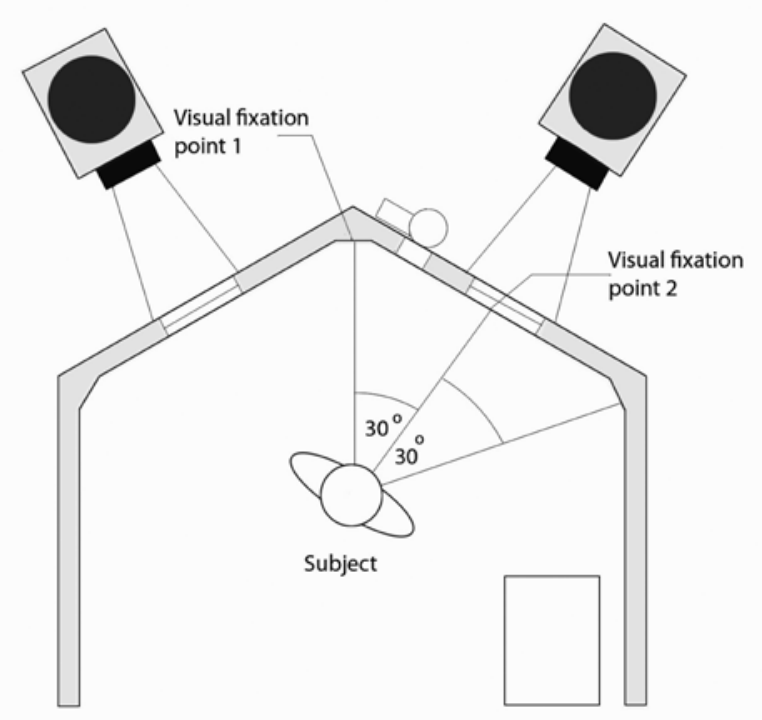

Figure 4: A view of the apparatus.

\section{Stimuli Variations and Selections of Screen Image Samples}

There were two steps for varying the characteristics of the image- complexity, mystery, legibility and coherence. The first step was choosing two images, one for a natural scene and one for an urban scene. The second step was lessening the degree of each factor in the images. There were four treatments, one for each level of the factors, which are, for example for the degree of complexity: 1) an image with high complexity (reference image), 2) an image with moderate complexity, 3) an image with low complexity, and 4) an image with no complexity (blank image or control treatment). The final 32 screen images, eight screen images for a study of each factor, were used in this experiment as shown below: 


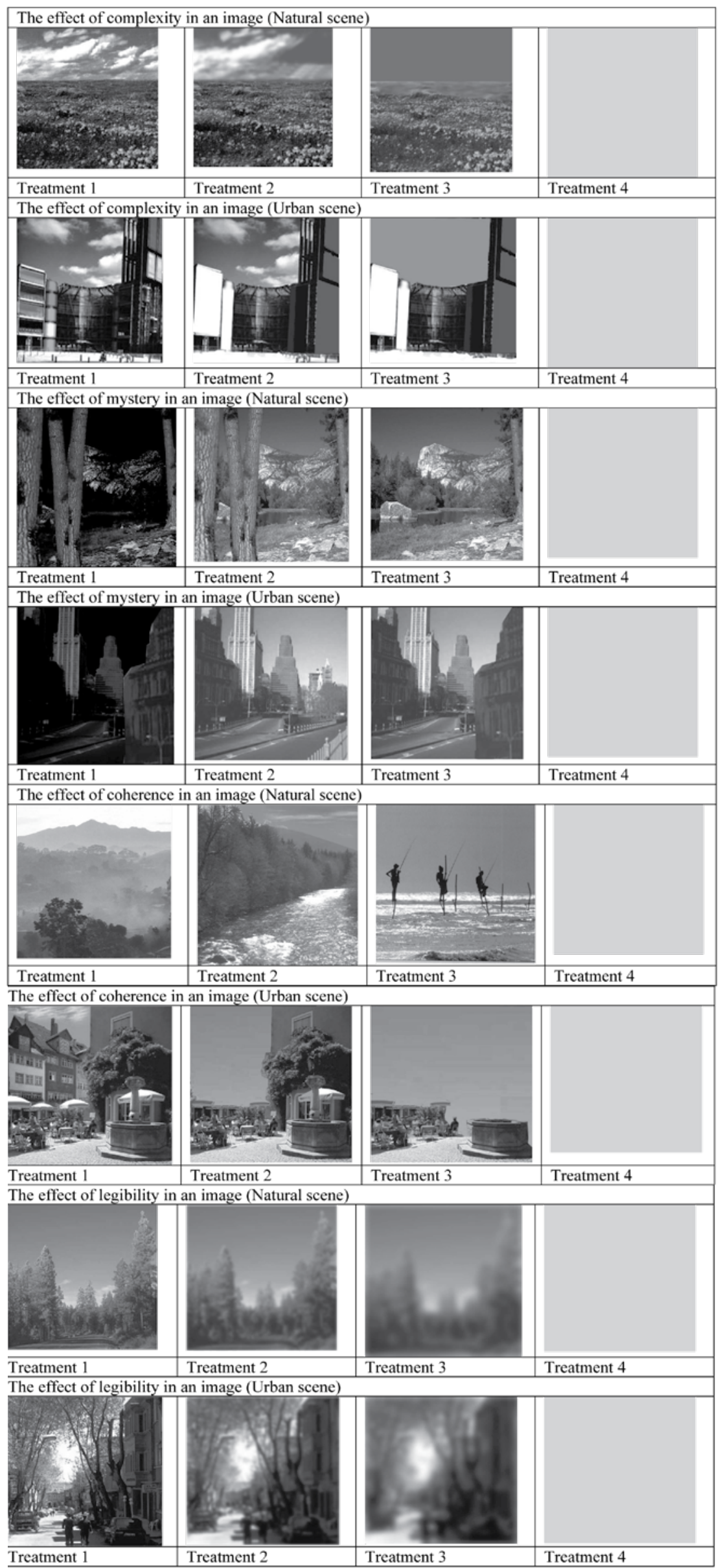

Figure 5: The thirty-two screen images used in the second experiment 


\section{Experimental Equipment and Measurements}

Source luminance was taken using an illuminance meter and background luminance was taken using a luminance meter. The source luminance was held constant at $12,000 \mathrm{cdm}-2$ in this experiment. The background luminance was held constant at $65 \mathrm{cdm}$ 2 . The values of the solid angle subtended at the observer's eyes by the glare source and the position of the source were also held constant. The area of each stimulus was $8 \mathrm{~cm} \times 8 \mathrm{~cm}$. with the distance from the centre of the stimulus to the eye being $0.60 \mathrm{~m}$.

\section{Experimental Procedures}

Twenty-eight subjects took part in this experiment consisting of males and females between 20 and 30 years of age. There was an equal balance between males and females and a balance between subjects with spectacles and without spectacles. Half of men and half of women wore spectacles. The subjects were recruited from students and other staff in $\mathrm{Na}$ resuan University. The experimental design was a repeated measures Balanced Latin Square design.
There were four pictures in each treatment. The same subjects experienced all treatments so that the effects of other variables from subjects would be minimized. All the stimulus images to be presented were randomly assigned to the subjects. There were two periods - a pretest and a real experimental period. The pretest period process was similar to the first experiment. After the period of the pretest, each subject was told to fix his/her eye position at the fixation point- the centre of the right screen. The first image was presented automatically and the subject was asked to rate perceived discomfort glare by marking on GSV scales. The subjects had to complete this process within three seconds for each stimulus until they finished all twenty-four screen images. Discomfort glare in this study was determined by a subjective glare rating using a continuous scale with four criteria called the Glare Sensation Vote (GSV) (Iwata and Tokura, 1998). A vote could be made by marking a tick at any point on the line of the continuous scale. The Glare Sensation Vote (GSV) value was defined as the value marked by subjects on this scale. For data analysis, numbers were assigned as follows:

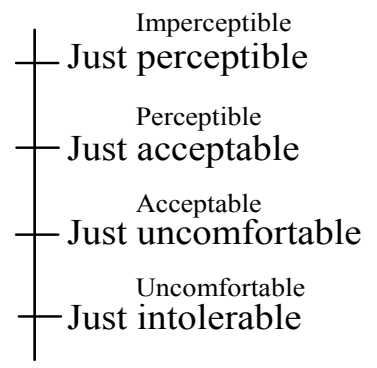

Figure 6: The Glare Sensation Vote (GSV) used in the subjective assessment of this experiment

Results

Table 2: Mean and standard deviation of glare assessments (GSV) from images with different degrees of stratification and the significance level ( $p$-value) of the ANOVA analysis

\begin{tabular}{lcc}
\hline Image categories & Mean & SD \\
\hline High complex image (L1) & 0.61 & 0.58 \\
Moderate complex image (L2) & 0.73 & 0.71 \\
Low complex image (L3) & 1.09 & 0.88 \\
Image with no complexity (L4) & 2.35 & 0.58 \\
$p$-value & $0.000^{* *}$ & \\
$* * \quad$ The mean difference is highly significant $(p r o b<0.01)$ in a one-way repeated measures ANOVA \\
$* \quad$ The mean difference is significant $(p r o b<0.05)$ in a one-way repeated measures ANOVA \\
NS No significant difference in a one-way repeated measures ANOVA
\end{tabular}

Table 3: Difference between mean glare assessment (GSV) from pairwise comparisons for images with different complexity

\begin{tabular}{lcccc}
\hline Image Categories & L1 & L2 & L3 & L4 \\
\hline L1 & 0.000 & & \\
L2 & 0.125 & 0.000 & \\
L3 & $0.491^{* *}$ & $0.366^{*}$ & 0.000 & \\
L4 & $1.743^{* *}$ & $1.618^{* *}$ & $1.252^{* *}$ & 0.000 \\
\hline ** & \multicolumn{2}{l}{ The mean difference is highly significant $($ prob $<0.01)$ in a Sidak $t$-test } \\
$*$ & \multicolumn{4}{l}{ The mean difference is significant $($ prob $<0.05)$ in a Sidak $t$-test }
\end{tabular}


The results, as can be seen in Table 2, show the effect of image complexity was highly significant $(p<0.01)$. Further analysis of multiple comparisons between means by the Sidak t-test, as can be seen from Table 3, indicates that images with high complexity and moderate complexity and images with low complexity are significantly less glaring than images with no complexity $(p<0.01)$. Similarly, it shows that images with high complexity are significantly less glaring than images with low complexity $(p<0.01)$. It can be seen that images with moderate complexity are less glaring than low complexity $(p<0.05)$.

Table 4: Mean and standard deviation of glare assessments (GSV) from images with different degrees of mystery and the significance levels ( $p$-value) of the ANOVA analysis

\begin{tabular}{lcc}
\hline Image categories & Mean & SD \\
\hline High mysterious image (L1) & 0.19 & 0.37 \\
Moderate mysterious image (L2) & 0.81 & 0.66 \\
Low mysterious image (L3) & 1.35 & 0.86 \\
Image with no mystery (L4) & 2.42 & 0.58 \\
$p$-value & $0.000^{* *}$ \\
\hline$* * \quad$ The mean difference is highly significant $($ prob<0.01) in a one-way repeated measures ANOVA \\
$* \quad$ The mean difference is significant (prob<0.05) in a one-way repeated measures ANOVA \\
NS No significant difference in a one-way repeated measures ANOVA
\end{tabular}

Table 5: Difference between mean of glare assessment (GSV) from pairwise comparisons for images with different mystery

\begin{tabular}{lcccc}
\hline Image Categories & L1 & L2 & L3 & L4 \\
\hline L1 & 0.000 & & \\
L2 & $0.616^{* *}$ & 0.000 & & \\
L3 & $1.153^{* *}$ & $0.537^{* *}$ & 0.000 & \\
L4 & $2.219^{* *}$ & $1.604^{* *}$ & $1.066^{* *}$ & 0.000 \\
** & The mean difference is highly significant (prob $<0.01)$ in a Sidak $t$-test \\
$*$ & The mean difference is significant (prob $<0.05)$ in a Sidak $t$-test
\end{tabular}

The results, as can be seen in Table 4 , show that the effect of image complexity was highly significant $(p<0.01)$. Further analysis of multiple comparisons between means by the Sidak t-test, as can be seen from Table 5, indicates that images with high mystery and moderate mystery and images with low mystery are significantly less glaring than images with no mystery $(p<0.01)$. Similarly, it shows that images with high mystery and moderate mystery are significantly less glaring than images with low mystery $(p<0.01)$. It can be seen that images with high complexity are less glaring than images with low mystery $(p<0.01)$.

Table 6: Mean and standard deviation of glare assessments (GSV) from images with different degrees of coherence and the significance levels ( $p$-value) of the ANOVA analysis

\begin{tabular}{lcc}
\hline Image categories & Mean & SD \\
\hline High coherent image (L1) & 0.42 & 0.47 \\
Moderate coherent image (L2) & 0.70 & 0.55 \\
Low coherent image (L3) & 1.44 & 0.62 \\
Image with no coherence (L4) & 2.55 & 0.40 \\
$p$-value & $0.000^{* *}$ \\
$* * \quad$ The mean difference is highly significant $(p r o b<0.01)$ in a one-way repeated measures ANOVA \\
$* \quad$ The mean difference is significant $($ prob $<0.05)$ in a one-way repeated measures ANOVA \\
NS No significant difference in a one-way repeated measures ANOVA
\end{tabular}

Table 7: Difference between mean of glare assessment (GSV) from pairwise comparisons for images with different coherence

\begin{tabular}{lcccc}
\hline Image Categories & L1 & L2 & L3 & L4 \\
\hline L1 & 0.000 & & \\
L2 & $0.280^{*}$ & 0.000 & & \\
L3 & $1.019 * *$ & $0.739 * *$ & 0.000 & \\
L4 & $2.127 * *$ & $1.846^{* *}$ & $1.107 * *$ & 0.000 \\
** & The mean difference is highly significant $($ prob $<0.01)$ in a Sidak $t$-test \\
$*$ & The mean difference is significant $($ prob $<0.05)$ in a Sidak $t$-test
\end{tabular}


The results, as can be seen in Table 6 , show that the effect of image coherence was highly significant $(p<0.01)$. Further analysis of multiple comparisons between means by the Sidak t-test, as can be seen from Table 7 , indicates that images with high coherence and moderate coherence and images with low coherence are significantly less glaring than images with no coherence $(p<0.01)$. Similarly, it shows that images with high coherence and moderate coherence are significantly less glaring than images with low coherence $(p<0.01)$. It can be seen that images with high coherence are less glaring than images with moderate coherence $(p<0.05)$.

Table 8: Mean and standard deviation of glare assessments (GSV) from images with different degrees of legibility and the significance levels ( $p$-value) of the ANOVA analysis

\begin{tabular}{lcc}
\hline Image categories & Mean & SD \\
\hline High legible image (L1) & 0.49 & 0.63 \\
Moderate legible image (L2) & 0.81 & 0.84 \\
Low legible image (L3) & 1.11 & 1.16 \\
Image with no legibility (L4) & 2.42 & 0.65 \\
$p$-value & $0.000 * *$ \\
$* * \quad$ The mean difference is highly significant $($ prob $<0.01)$ in a one-way repeated measures ANOVA \\
$* \quad$ The mean difference is significant $($ prob $<0.05)$ in a one-way repeated measures ANOVA \\
NS No significant difference in a one-way repeated measures ANOVA
\end{tabular}

Table 9: Difference between mean of glare assessment (GSV) from pairwise comparisons for images with different legibility

\begin{tabular}{lcccc}
\hline Image Categories & L1 & L2 & L3 & L4 \\
\hline L1 & 0.000 & & \\
L2 & 0.321 & 0.000 & & \\
L3 & $0.623^{* *}$ & 0.302 & 0.000 & \\
L4 & $1.927^{* *}$ & $1.605^{* *}$ & $1.304 * *$ & 0.000 \\
\hline$*$ & The mean difference is highly significant $($ prob $<0.01)$ in a Sidak $t$-test \\
$*$ & The mean difference is significant $($ prob $<0.05)$ in a Sidak $t$-test
\end{tabular}

The results, as can be seen in Table 8 , show the effect of image legibility was highly significant $(p<0.01)$. Further analysis of multiple comparisons between means by the Sidak t-test, as can be seen from Table 9 , indicates that images with high legibility and moderate legibility and images with low legibility are significantly less glaring than images with no legibility $(p<0.01)$. Similarly, it shows that images with high coherence are significantly less glaring than images with low coherence $(p<0.01)$. It can be concluded that the four factorscomplexity, mystery, coherence, and legibility in an image - have a significant effect on glare discomfort.

\section{Experiment III: The Effect of the Preferred View on Glare}

\section{Introduction}

Due to the time-constraints and the availability of views, only two factors, view complexity and mystery were investigated in terms of their effect on glare. This last experiment examined the effect of complexity in views and the effect of mystery within the scene. Subjects assessed glare in four settings, combinations of high and low scores on each factor.

\section{Research Methodology Experimental Setting}

The experiments were carried out in the Faculty of Architecture, Naresuan University, Figure 7. This building has rooms which are similar in size and internal finishes, but which face in different directions and are at different heights above ground. The scene from the windows varied with orientation, ranging from a hard urban view to trees and parkland, and at storey level from nearby objects at ground level to a broad distant view from sixth floor windows. We selected rooms with different types of view, and then set up an experimental layout in each, making the rooms identical except for the view. Figure 8 illustrates one of the rooms. Each test room was $4.00 \mathrm{~m}$ deep by $3.00 \mathrm{~m}$ wide and $3.00 \mathrm{~m}$ high, and had four tall windows on one side. One of these was used as the test window; the others were covered with fully closed Venetian blinds, thick opaque paper covering any gaps. The blind in front of the test window was partly closed, leaving an aperture $0.80 \mathrm{~m}$ wide and $1.00 \mathrm{~m}$ high. The ceiling of each room was matt white with reflectance ${ }_{c}=0.8$, the walls $\square_{w}=0.6$, and the floor $\square_{f}=0.2$. The subjects sat facing the window $2 \mathrm{~m}$ from the plane of the glass. There were two experimenters who stood slightly behind the subject at each side. One experimenter operated a digital camera, recording the view at a range of sensitivities. These images would later be used to derive the luminance distribution of the scene. The monitor- 
ing procedures followed the method proposed in the IEA SHC Task 21 (Coutelier, 2002; Velds, 2000). A further illuminance meter recorded the vertical illuminance on a tilted plane at the subject's position.

\section{Experimental Procedures}

In the preliminary test, sixteen real views were observed in real rooms by twenty university students in architecture but differing in nationality and social background. The subjects were asked to assess the degree of complexity and mystery using questionnaires with five-point rating scales. The views were ranked by mean score.

There were two periods in this experiment: 1) the presetting-up period and 2) the real test. In the presetting-up period, on arrival at the test room, the subject was asked to sit in the test position facing the window. The experimenter explained the purpose of the study, and the subject completed a consent form. The subject was then asked to fill out a questionnaire covering gender, age, whether he or she was a student or had a lighting or architecture background, used glasses or contact lenses, had colour blindness or other eye problems. There was also an open ended question that enabled the subject to note any other points. The subject then read a sheet of instructions. These contained a definition of glare, the meaning of the criteria, and the procedure for making evaluations. The experimenter demonstrated the procedure, choosing an arbitrary value (this was recorded so a check could

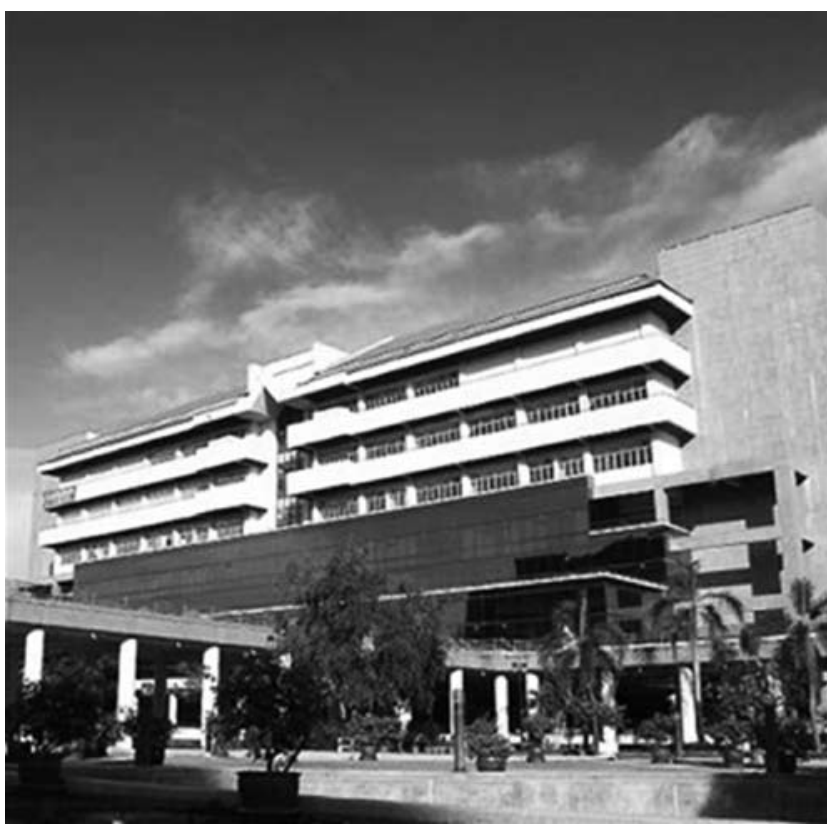

Figure 7: Faculty of Architecture, Naresuan University be made of any possible influence on the subject's choices). The subject then made five evaluations of discomfort glare using this procedure at 30 second intervals. There was a relaxation period of about two minutes before the actual test was made. In the real test, the subject was seated at a chair. When instructed, he or she looked up towards the window and focussed on the centre of the opening for 30 seconds. The experimenter then asked the subject to record the glare level by making a mark on the GSV scale on the questionnaire form and to say 'yes' when this was done, as a signal to record light values and to take photographs.

Each subject viewed one of four test conditions: 1) the low complex and low mysterious view, 2) the low complex and high mysterious view, 3) the high complex and low mystery view, and 4) the high complex and high mysterious view (See Figure 9). The subjects were allocated to the views randomly. The two views are shown (bottom-right and top-left) in Figure 3.The Hopkinson Cornell Daylight Glare Index (DGI) was calculated from the luminous and angular values measured when each subject assessed glare discomfort. We used this equation because it is the most cited window glare formula. The Glare Sensation Vote (GSV) was derived from each subject's questionnaire, and the Glare Response Vote (GRV) calculated, as above. The main statistical methods used were Twoway Analysis of covariance (ANCOVA) and Sidak ttest to examine the significance of difference between glare discomfort under different test conditions.

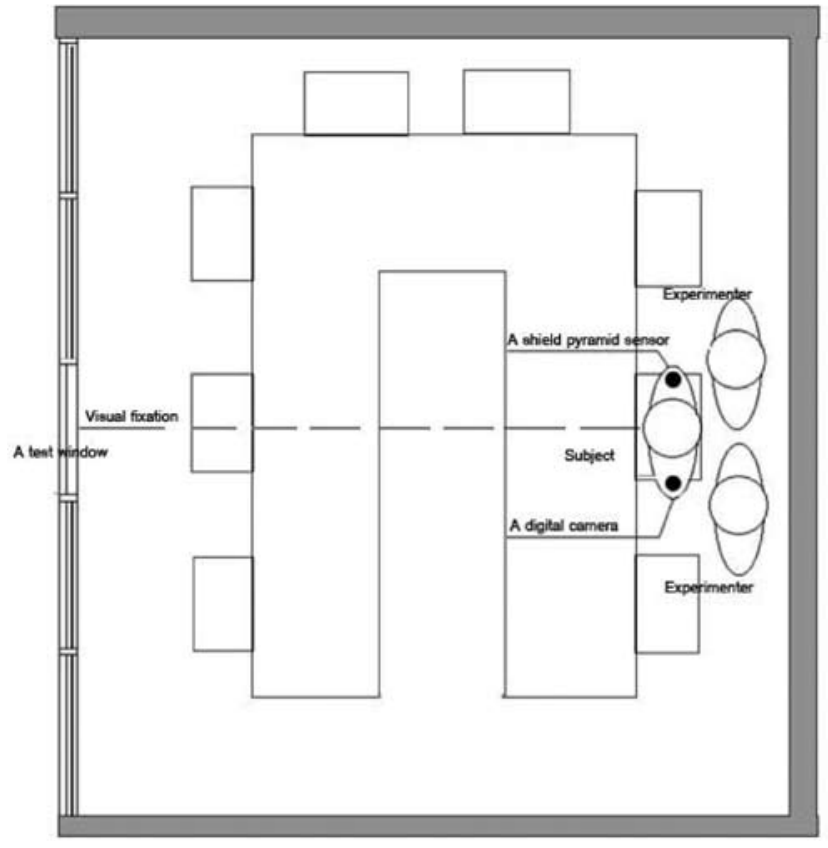

Figure 8: One of the rooms used in the first experiment 

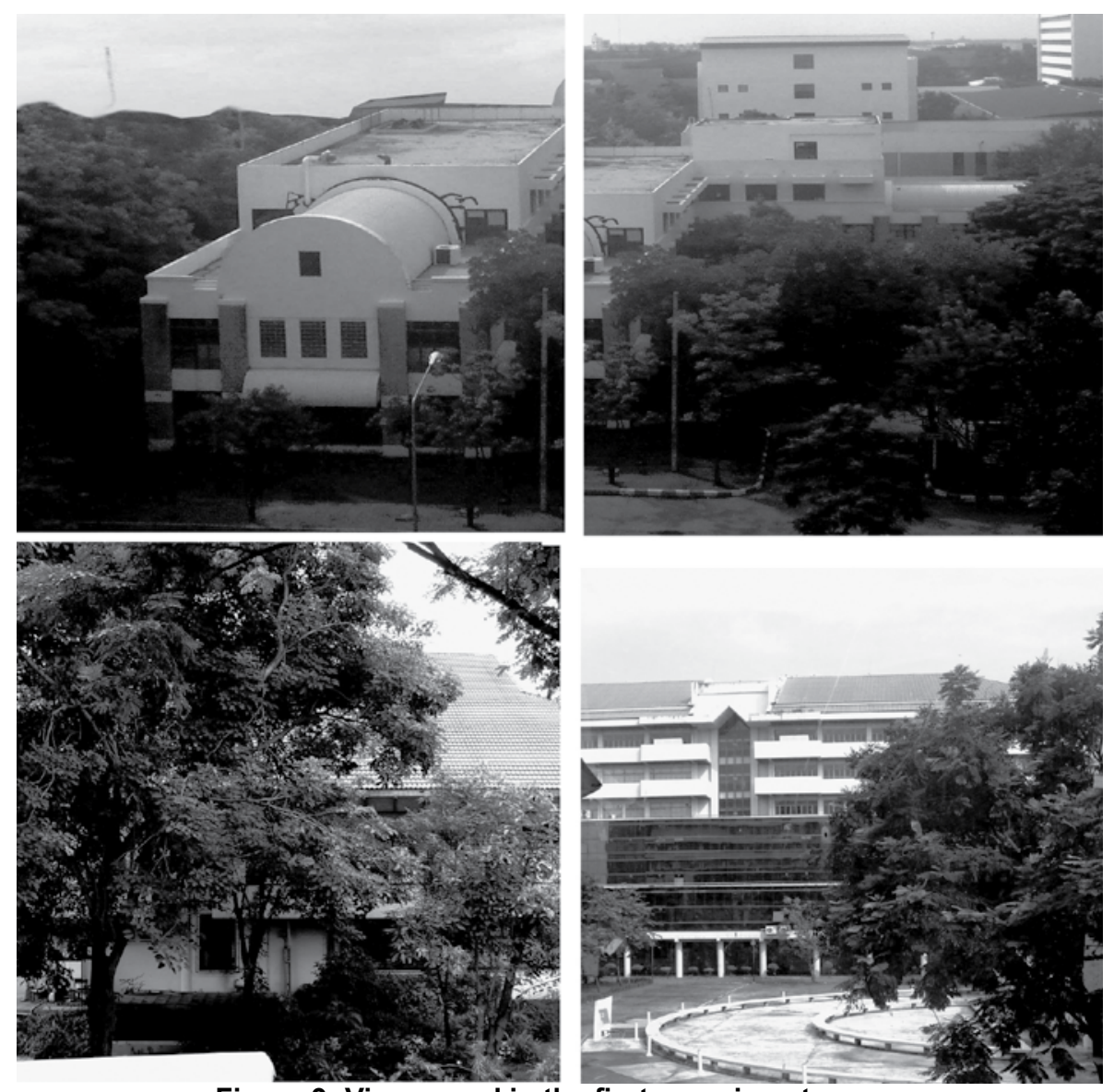

Figure 9: Views used in the first experiment

\section{Results}

Figure 10 shows the relationships between DGI and GRV for the four cases. Two-way ANCOVA revealed no interaction effect between the factors (high complex: low complex, high mysterious : low mysterious) but showed that the high complex views are significantly less glaring than the low complex views $(p<0.01)$, and that the high mysterious views are significantly less glaring than the low mysterious views $(p<0.01)$.

The Sidak t-test was used to compare the assessments between each treatment: the low complex and low mysterious view is significantly more glaring than the others $(p<0.01)$, and the urban three-layer view is significantly less glaring than the urban one-layer view $(p<0.01)$. The test also showed that the low complex and high mysterious view is significantly more glaring than the high complex and low mysterious view $(p<0.05)$ and the high complex and high mysterious view $(p<0.01)$. However, no significant difference was found between the high complex and low mysterious view and the high complex and high mysterious view.

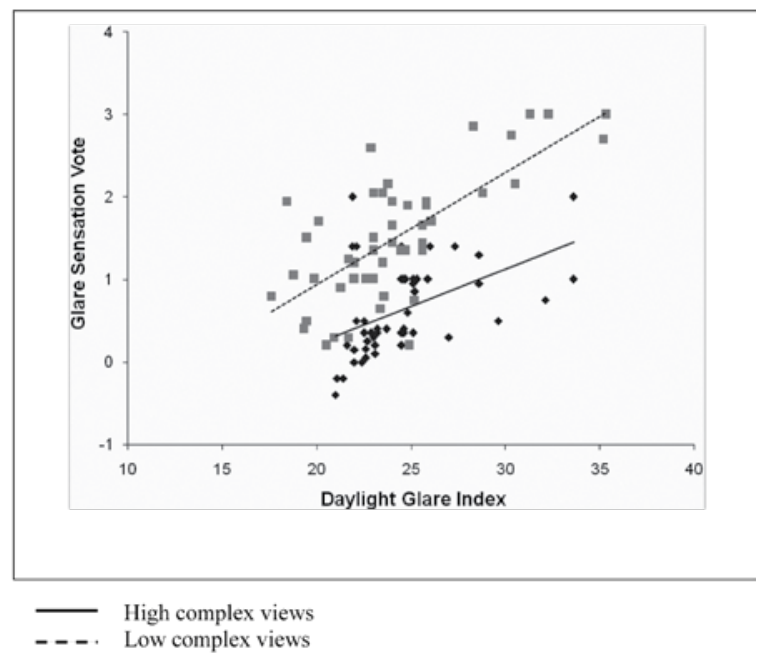

Figure 10: Daylight Glare Index (DGI) calculated versus Glare Response Vote (GRV) judged by subjects for high complex views and low complex views. The horizontal axis represents the calculated Daylight Glare Index, the vertical axis represents the Glare Response Vote reported by subjects or GRV. $\checkmark$ refers to high complex views, $\square$ refers to low complex views. The lines are trend lines of the fitted function. 


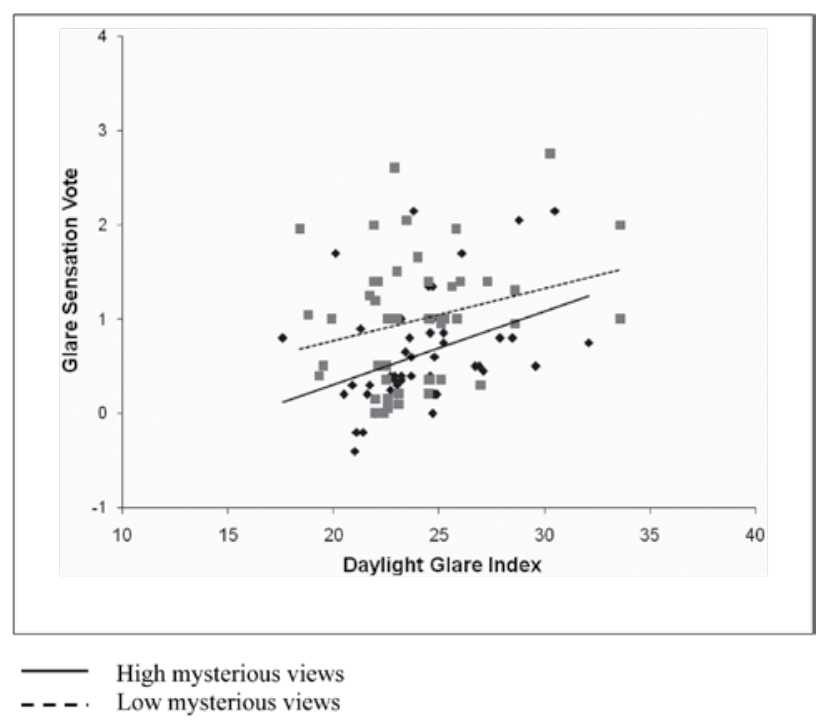

Figure 11: Daylight Glare Index (DGI) calculated versus Glare Response Vote (GRV) judged by subjects for views with high mysterious and those with low mysterious. $\nabla$ refers to high mysterious views. $\square$ refers to low mysterious views. The lines are trend lines of the fitted function.

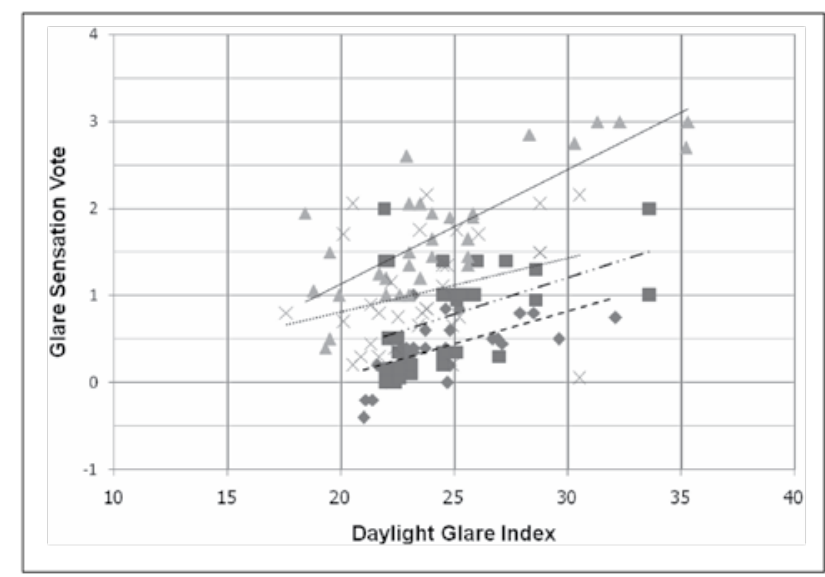

_ low complex and low mysterious view
$-\cdots$$\quad \begin{aligned} & \text { high complex and low mysterious view } \\ & -\cdots\end{aligned}$

Figure 12: Daylight Glare Index (DGI) calculated versus Glare Response Vote (GRV) judged by subjects for four views. $\Delta$ refers to low complex and low mysterious view, $X$ represents high complex and low mysterious view. $\square$ refers to low complex and high mysterious. $\checkmark$ represents high complex and high mysterious view. The lines are trend lines of the fitted function.

\section{Discussion and Conclusion}

The result showed that the presence of view influences the degree of discomfort glare. It also showed that discomfort glare is dependent on image stratification and suggested the significant effect of four factors relating to the theory of the landscape preference matrix-complexity, mystery, coherence and legibility. The results of this study confirm the importance of view outside on discomfort glare reduction and are consistent with the work of some prior investigators.

An explanation of the effect of image on discomfort glare sensation has come from some evidence of prior investigators. Earlier studies have also found that the presence of foreground in the landscape scene may strongly influence the preference of observers (Ulrich, 1977; Wohwill, 1973). According to Gibson (1958), the characteristics of ground texture profoundly affect the accuracy of depth estimates. Specifically, it can play a very important role in defining depth and helping the observers to comprehend element relationships in three dimensions (Ulrich, 1977). In this way, more information can be extracted and, therefore, influence the interests and/or pleasure of observers. Based on their characteristics, images with three layers referred the clearest three-dimensional space of the landscape. Thus, they provided more information that could attract the interest of the subjects. On the other hand, when depth in the images is restricted or 
could not be perceived, the scenes seemed to stand ambiguously in two dimensions and appraisal was also essentially limited. Accordingly, images with one layer, either sky or ground, with the most restricted depth appeared to be least influential on discomfort glare sensation.

The results of the effect on glare of image complexity, mystery, coherence and legibility are also consistent with other prior studies in Environmental Psychology (Berlyne, 1971; Kaplan and Kaplan, 1995). Rappoport and Kantor (1967 p210) claimed that "High complexity can hold much of the attention of the perceivers." Gesell et al. (1949 in Kahneman, 1973) also pointed out that novelty and complexity are important collative properties that control spontaneous attention in the adult. Based on these, it could be implied that window-views containing an appropriate level of these factors could optimize discomfort glare.

It is also necessary to note some limitations specific to this experiment that affect interpretation of the results. The appraised glare discomfort in this study was limited to only one particular group of subjects. Moreover, the setting was artificial; extensions of the results are contingent on the stimuli and the experimental conditions considered in the study.

\section{Acknowledgement}

The author would like to thank the Thailand Research Fund for funding this project.

\section{Reference}

Berlyne, D. E., 1971. Aesthetics and Psychobiology, New York: Appleton-Century-Crofts.

Berman, S.M., Bullimore, M.A., Bailey, I.L.,

Jacobs, R.J., 1996. The Influence of Spectral Com position of Discomfort Glare for Large-size Sources. Journal of Illuminating Engineering Society, pp. 34-41.

Boyce, P.R., 1981. Human Factors in Lighting. Ap plied Science Publishers Ltd. London.

Coutelier, B., 2002. Luminous environment descrip tors: proposals based on field observations. IEA Task 31 and CIE Division 3 mini-confer ence - NRC-IRC. Ottawa. Canada

Gibson, J. J., 1958. Perception of Distance and Space in the Open Air. In Beardslee, D., and Wertheimer, M. (Eds), Readings in Per ception. Princeton: Van Nostrand, pp 415431.
Iwata, T., and Tokura, M., 1998. Examination of limi tations of predicted glare sensation vote (PGSV) as a glare source- Towards a com prehensive development of discomfort glare evaluation. Lighting Research and Technol ogy, 30, pp 81-88.

Kahneman, D., 1973. Attention and effort. Prenticehall Series in Experimental Psychology. New Jersey.

Kaplan, S., and Kaplan, R., 1995. The Experience of Nature: A Psychological Perspective. Cam bridge: Cambridge University Press.

Keighley, E. C., 1973. Visual Requirements and Re duced Fenestration in Offices: A Study of Window Shape. Building Science, 8, pp 311320.

Lynes, J. A., 1974. The Window as a Communica tion Channel. Light and lighting, pp 284-288.

Markus, T. A., 1967a. The Significance of Sunshine in Office Workers. Proceeding CIE Confer ence on "Sunlight on Building". Bowcentrum. Rotterdam, pp 59-93.

Markus, T. A., 1967b. The Functions of Windows- A Reappraisal. Building Science, 2, pp 97-121.

Osterhaus, W.K.E, 1998, Brightness as Simple In dicator for Discomfort Glare from Large Area Glare Sources, Proceeding of the First CIE Symposium on Lighting Quality, Ottawa, Can ada, May 9-10.

Rappoport, A. and Kantor, R.E., 1967, Complex ity and ambiguity in Environmental Design, Journal of American Institute of Planners, Vol.23, p.210-221.

Tuaycharoen, N., Tregenza, P.R., 2005, Discom fort glare from interesting images, Lighting Research and Technology, Vol.37, No.4, pp.329- 341.

Tuaycharoen, N., 2006, The reduction of discomfort glare from windows by interesting views, PhD thesis, Sheffield: The University of Shef field.

Ulrich, R. S., 1977, Visual Landscape Preference: A Model and Application, Man-Environment Systems, Vol. 7, pp. 279-293.

Velds, M. 2000. Assessment of lighting quality in of fice rooms with daylighting system. PhD Thesis. Delft University of Technology. Delft. Netherlands.

Wohwill, J. F., 1973, Factors in Differential Re sponse to The Natural and Man-Made Envi ronments, Paper Presented At The Annual Meeting of The American Psychological As sociation, Montreal, August. 